\title{
Controle do vigor em pereira 'Abate Fetel' por meio da poda radicular
}

\author{
Vigor control in 'Abate Fetel' pear by root pruning
}

\author{
Douglas André Würz $^{1 \star}$, Mayra Juline Gonçalves ${ }^{2}$, Rafael Daboit ${ }^{3}$, Leo Rufato ${ }^{2}$ e Aike Anneliese \\ Kretzschmar $^{2}$
}

\author{
${ }^{1}$ Instituto Federal de Santa Catarina, Canoinhas, SC, Brasil. *Autor para correspondência: douglaswurz@hotmail.com. \\ ${ }^{2}$ Universidade do Estado de Santa Catarina, Lages, SC, Brasil. \\ ${ }^{3}$ Profissional Autônomo, Lages, SC, Brasil.
}

Submissão: 29/10/2018 / Aceite: 01/03/2019

\begin{abstract}
RESUMO
O objetivo deste trabalho foi avaliar o efeito de diferentes níveis de poda radicular sobre o crescimento vegetativo, produção e qualidade de frutos de pereira Abate Fetel. $O$ experimento foi conduzido durante as safras 2007 e 2008. Os tratamentos consistiram em: poda radicular a $20 \mathrm{~cm}$ da planta, $35 \mathrm{~cm}$ e controle sem realização de poda radicular. A poda radicular apresentou controle do vigor e na manutenção de características vegetativas, no entanto, não influenciou os índices produtivos e físicoquímicos dos frutos. Conclui-se que a poda radicular promove controle do vigor em pereira Abate Fetel.
\end{abstract}

PALAVRAS-CHAVE: Pyrus communis L., Controle do crescimento, produção de frutos.

\begin{abstract}
The objective of this work was to evaluate the effect of different levels of root pruning on the vegetative growth, yield and quality of fruits of 'Abate Fetel' pear. The experiment was conducted during the cropping seasons of 2007 and 2008. The treatments consisted of: root pruning at $20 \mathrm{~cm}$ of the plant, $35 \mathrm{~cm}$ and control without root pruning. Root pruning showed vigor control and maintenance of vegetative characteristics, however, it did not influence the productive and physical-chemical indices of the fruits. It is concluded that root pruning promotes vigor control in 'Abate Fetel' pears.
\end{abstract}

KEYWORDS: Pyrus communis L., growth control, fruit production.

A pera (Pyrus spp.) é a fruta mais importada no Brasil, de acordo com a FAO (2016). De acordo com CARRA et al. (2017a), a pereira é uma das frutíferas menos produtivas no Brasil. Entre os motivos para a baixa produtividade, HAWERROTH et al. (2012), atribui a baixo número de frutos e o longo período juvenil às condições climáticas, favorecendo um maior crescimento vegetativo. Em estudos sobre o hábito de frutificação e produção de pereiras em diferentes porta-enxertos, observou-se que a formação de flores, estruturas de produção e eficiência produtiva é inversamente proporcional ao vigor (PASA et al. 2011).

O controle do crescimento vegetativo é necessário para evitar crescimento excessivo do dossel, evitando o sombreamento, proporcionando maior intercepção de radiação solar e melhorando a qualidade dos frutos (RADEMACHER 2004). Além disso, o crescimento vegetativo excessivo tem um efeito negativo na produtividade (PASA et al. 2011). Dentre as técnicas de controle de vigor destacam-se arqueamento de ramos, poda e uso de porta-enxerto anão. No entanto, CARRA et al. (2017b), destaca que a poda de inverno e verão podem elevar muito os custos de produção, sendo assim, outras alternativas de controle de vigor são necessárias, como o uso de reguladores de crescimento e a poda radicular, a fim de proporcionar equilíbrio adequado entre crescimento vegetativo e frutificação.

Embora a poda radicular seja considerada uma opção eficaz para a redução de vigor (CARRA et al. 2017b, FACHINELLO \& ROBINSON 2017), faz-se necessário definir e controlar a severidade, profundidade da poda e distância do tronco, a fim de otimizar seu desempenho. Nesse contexto, este trabalho teve como objetivo avaliar o efeito da poda radicular no controle do vigor, produção e qualidade de frutos de pereira europeia cultivar Abate Fetel.

O experimento foi conduzido em pomar comercial de pereira europeia, localizada no município de Vacaria, estado do Rio Grande do Sul, durante as safras agrícolas de 2006/07 e 2007/08. O pomar está 
localizado a $980 \mathrm{~m}$ de altitude, em coordenadas $50^{\circ} 42^{\prime} \mathrm{W}$ e $28^{\circ} 33^{\prime} \mathrm{S}$.

O pomar foi implantado em 2000 com plantas da cultivar Abate Fetel enxertadas sobre marmelo 'Adams'. O plantio foi realizado no sentido leste-oeste, em espaçamento de $70 \mathrm{~cm}$ entre plantas e $3 \mathrm{~m}$ entre linhas e as plantas conduzidas no sistema de líder central.

A poda radicular foi executada de agosto de 2006, com o auxílio de uma lâmina de corte acoplada ao sistema hidráulico de um trator. Os tratamentos consistiram em: poda radicular a $20 \mathrm{~cm}$ da planta, poda radicular a $35 \mathrm{~cm}$ da planta e controle sem realização de poda radicular. O corte radicular foi efetuado a uma profundidade média de 30 a $40 \mathrm{~cm}$ a partir do nível de solo.

As variáveis analisadas foram: área de secção transversal do tronco (ASTT) (obtida utilizando a fórmula da área de uma circunferência $\left(\pi r^{2}\right)$ onde $r$ é o raio, obtido a partir da metade do diâmetro médio do tronco); comprimento médio de ramos (determinado através da medida de cinco ramos aleatórios de cada planta, medido com auxílio de fita métrica, dados expressos em $\mathrm{cm}$ ); volume de copa (obtido pelas medidas de largura, espessura e altura da copa, medida a partir da inserção do primeiro ramo basal do tronco, com o auxílio de uma fita métrica, e dados expressos em $\mathrm{m}^{3}$ ); número médio de gemas (determinado através de avaliação em cinco ramos demarcados nas plantas, afim de se estabelecer a relação entre número de gemas e comprimento de ramos).

$\mathrm{Na}$ época da colheita, no mês de fevereiro, os frutos foram colhidos, pesados e encaminhados ao Núcleo de Tecnologia de Alimentos do Centro de Ciências Agroveterinárias da Universidade do estado de Santa Catarina (NUTA - CAV/UDESC) para avaliação e determinação do teor de sólidos solúveis (SS) (determinado por refratômetria, utilizando gotas de suco puro de cada repetição, expressando-se o resultado em ${ }^{\circ}$ Brix); firmeza da polpa das frutas (determinada utilizando um penetrômetro manual, com ponteira plana de $8 \mathrm{~mm}$ de diâmetro, sendo efetuadas duas leituras por fruta, em lados opostos da região equatorial, após a remoção de uma pequena e superficial porção da casca, sendo os resultados expressos em Newtons (N)); acidez total (AT), determinada pela titulação potenciométrica com $\mathrm{NaOH} 0,1 \mathrm{~N}$. Os resultados foram expressos em \% de acidez. Para cada avaliação foram utilizadas quatro repetições com dez frutos.

Os tratamentos foram dispostos a campo em blocos casualizados, sendo quatro blocos com seis plantas, onde foram avaliadas as duas plantas centrais, sendo o delineamento utilizado em campo o mesmo utilizado no laboratório. Os dados obtidos foram submetidos à análise de variância e, quando significativos, as médias foram comparadas pelo teste Tukey a $5 \%$ de probabilidade de erro.

No primeiro ano de avaliação não houve diferença significativa entre os tratamentos para a variável área da seção transversal do tronco (ASTT), no entanto no segundo ano de avaliação, o tratamento com poda radicular a $35 \mathrm{~cm}$ diferiu dos demais tratamentos. (Tabela 1). Para a variável comprimento de ramos, os tratamentos diferiram significativamente. Na primeira safra, os tratamentos com a poda de raiz apresentam maior comprimento de ramos, no entanto, na segunda safra, o tratamento testemunha apresentaram o maior valor para comprimento de ramos, diferindo dos demais tratamentos (Tabela 1). Assim, verificou-se que o uso da poda radicular, nas duas intensidades, mostrou-se eficiente para controlar o crescimento dos ramos. VERCAMMEN et al. (2005) e WANG et al. (2014) verificaram que plantas utilizadas em experimentos com poda radicular apresentaram redução em altura, na segunda aplicação desta técnica, além de redução no comprimento, número e diâmetro dos brotos.

Com relação ao volume de copa, no primeiro ano de avaliação não houve diferença significativa entre os tratamentos. No segundo ano, os tratamentos diferem entre si, sendo o tratamento com poda de raiz a 20 $\mathrm{cm}$ de distância da planta o que apresentou menor valor de volume de copa. De acordo com os resultados do trabalho de YEHIA et al. (2014), o qual efetuaram poda de raiz a 30, 60 e $90 \mathrm{~cm}$ em ambos os lados da planta, todos os tratamentos diminuíram o volume da parte aérea quando comparado ao tratamento sem a poda, o qual manteve o padrão de crescimento inalterado.

A redução do crescimento vegetativo, ocasionada pela poda radicular podem estar relacionadas a uma menor absorção de água (JANSSENS et al. 2011) e nutrientes (WANG et al. 2014) pela planta, devido ao reduzido volume de solo ocupado pelas raízes. Além disso, as raízes são o principal local de síntese de citocinina, hormônio vegetal responsável para divisão celular e alongamento, que desempenha um papel importante na promoção de crescimento vegetativo. Portanto, a redução do volume da raiz reduziria os níveis de citocininas transportados para o dossel, limitando assim seu crescimento (CARRA et al. 2017b).

Para a variável número de gemas, não foi verificada diferença entre os tratamentos em ambos os anos de avaliação. Para a relação número gemas/comprimento de ramos, na primeira safra, o tratamento testemunha apresentou diferença significativa dos demais tratamentos, sendo superior a estes. No segundo ano, estes valores se alteraram, e os níveis da poda radicular se diferem da testemunha, apresentando 
valores superiores a esta, comprovando os efeitos positivos na utilização da poda radicular (Tabela 1).

Tabela 1. Área da seção transversal do tronco (ASTT), comprimento de ramos, número de gemas, número gemas/comprimento, número de frutos, ASTT/número de frutos, volume de copa/número de frutos, número de frutos* peso, eficiência produtiva por plantas de pereira europeia Abate Fetel com o uso de diferentes tipos de poda radicular na safra agrícola de 2006/2007 e 2007/2008.

Table 1. Trunk cross-sectional area (ASTT), length of branches, number of buds, number of buds / length, number of fruits, ASTT/number of fruits, crown volume/number of fruits, number of fruits * productive efficiency by European pear plants Abate Fetel with the use of different types of root pruning in the 2006/2007 and 2007/2008 agricultural crops.

\begin{tabular}{|c|c|c|c|c|c|c|c|c|c|}
\hline \multirow[b]{2}{*}{ Tratamento } & \multicolumn{6}{|c|}{ Safra 2006/2007 } & & & \\
\hline & $\begin{array}{l}\text { ASTT } \\
\left(\mathrm{cm}^{2}\right)\end{array}$ & $\begin{array}{c}\text { Comprimento } \\
\text { de ramos } \\
\left(\mathrm{m}^{3}\right)\end{array}$ & $\begin{array}{l}\text { Volume } \\
\text { de copa } \\
\qquad\left(\mathrm{m}^{3}\right)\end{array}$ & $\begin{array}{l}\text { Número } \\
\text { de } \\
\text { gemas }\end{array}$ & $\begin{array}{l}\text { Número } \\
\text { gemas/ } \\
\text { comp. } \\
\text { ramos }\end{array}$ & $\begin{array}{l}\text { Número } \\
\text { de frutos }\end{array}$ & $\begin{array}{c}\text { ASTT } \\
\text { /número } \\
\text { de frutos }\end{array}$ & \multicolumn{2}{|c|}{$\begin{array}{l}\text { Volume de } \\
\text { copa/número } \\
\text { de frutos }\end{array}$} \\
\hline Testemunha & $26,7 \mathrm{a}$ & $23,9 \mathrm{~b}$ & $3,05 a$ & $17,4 \mathrm{a}$ & $0,72 a$ & $15,4 a$ & $1,73 a$ & \multicolumn{2}{|c|}{$0,19 a$} \\
\hline $20 \mathrm{~cm}$ & $28,2 \mathrm{a}$ & $30,6 \mathrm{a}$ & $3,69 a$ & $16,5 \mathrm{a}$ & $0,53 \mathrm{~b}$ & $16,2 \mathrm{a}$ & $1,74 \mathrm{a}$ & \multicolumn{2}{|c|}{$0,22 \mathrm{a}$} \\
\hline $35 \mathrm{~cm}$ & $30,4 \mathrm{a}$ & $30,6 \mathrm{a}$ & $3,77 \mathrm{a}$ & $16,5 \mathrm{a}$ & $0,53 b$ & $15,7 \mathrm{a}$ & $1,93 a$ & \multicolumn{2}{|c|}{$0,24 a$} \\
\hline Médias & 28,46 & 28,39 & 3,5 & 16,83 & 0,59 & 15,76 & 1,79 & \multicolumn{2}{|c|}{0,21} \\
\hline CV (\%) & 16,37 & 11,82 & 21,14 & 8,62 & 13,8 & 28,78 & 29,8 & \multicolumn{2}{|c|}{31,1} \\
\hline \multicolumn{10}{|c|}{ Safra $2007 / 2008$} \\
\hline Tratamento & $\begin{array}{l}\text { ASTT } \\
\left(\mathrm{cm}^{2}\right)\end{array}$ & $\begin{array}{l}\text { Comprimento } \\
\text { de ramos }(m)\end{array}$ & $\begin{array}{c}\text { Volume } \\
\text { de copa } \\
\left(\mathrm{m}^{3}\right)\end{array}$ & $\begin{array}{l}\text { Número } \\
\text { de } \\
\text { gemas }\end{array}$ & $\begin{array}{l}\text { Número } \\
\text { gemas/ } \\
\text { comp. } \\
\text { ramos }\end{array}$ & $\begin{array}{l}\text { Número } \\
\text { de frutos }\end{array}$ & $\begin{array}{l}\text { ASTT/ } \\
\text { número } \\
\text { de frutos }\end{array}$ & $\begin{array}{c}\text { Volume } \\
\text { de } \\
\text { copa/ } \\
\text { número } \\
\text { de } \\
\text { frutos }\end{array}$ & $\begin{array}{l}\text { Eficiên- } \\
\text { cia } \\
\text { prod } \mathrm{Kg} \\
\mathrm{cm}^{-2} \text {. }\end{array}$ \\
\hline Testemunha & $36,3 b$ & $59,6 \mathrm{a}$ & $5,03 \mathrm{a}$ & $17,4 \mathrm{a}$ & $0,29 \mathrm{~b}$ & $14,8 \mathrm{a}$ & $2,45 \mathrm{a}$ & $0,28 \mathrm{a}$ & $27,0 \mathrm{a}$ \\
\hline $20 \mathrm{~cm}$ & $37,6 \mathrm{~b}$ & $41,5 b$ & $4,04 \mathrm{~b}$ & $16,5 \mathrm{a}$ & $0,39 a$ & $16,3 \mathrm{a}$ & $2,30 \mathrm{a}$ & $0,24 a$ & $10,2 b$ \\
\hline $35 \mathrm{~cm}$ & $42,9 a$ & $40,5 b$ & $4,98 \mathrm{a}$ & $16,5 \mathrm{a}$ & $0,40 a$ & $13,7 \mathrm{a}$ & $3,13 a$ & $0,36 \mathrm{~b}$ & $14,4 \mathrm{~b}$ \\
\hline Médias & 38,9 & 47,2 & 7,81 & 16,8 & 0,36 & 14,9 & 2,62 & 0,29 & 17,3 \\
\hline CV (\%) & 11,2 & 12 & 4,68 & 8,62 & 16,1 & 22,68 & 25,5 & 14,2 & 31,6 \\
\hline
\end{tabular}

Médias seguidas da mesma letra na coluna não apresentam diferença estatística pelo teste de Tukey a $5 \%$ de probabilidade de erro.

O número de frutos não diferiu significativamente nas safras avaliadas, o mesmo ocorreu para a relação ASTT/número de frutos. Para a relação volume de copa/número de frutos, no primeiro ano de avaliação, não houve diferença estatística entre os tratamentos, no entanto no segundo ano, esta relação foi significativa. A testemunha não diferiu significativamente da poda de raiz a $35 \mathrm{~cm}$, e estas duas diferiram da poda a $20 \mathrm{~cm}$. Resultado semelhante também foram observados por MARSAL et al. (2002).

Para a variável número de frutos/massa, houve diferença significativa, onde a poda a $20 \mathrm{~cm}$ apresentou o maior valor, 3,85, seguido da poda a $35 \mathrm{~cm}$, com 3,02 e em terceiro nível a testemunha com 1,45 (Tabela 1). A eficiência produtiva, apresentou diferença significativa entre os tratamentos. A testemunha diferiu dos demais tratamentos, apresentando eficiência de $27 \mathrm{Kg} \mathrm{cm}^{-2}$, enquanto que com a poda a $20 \mathrm{~cm}$ a eficiência foi de 10,2 e na poda a $35 \mathrm{~cm}, 14,4 \mathrm{Kg} \mathrm{cm}^{-2}$. A poda radicular apesar de demonstrar resultados positivos no controle do vigor não influenciou significativamente nos índices produtivos. Resultados semelhantes foram observados por CARRA et al. (2017b). ASÍN et al. (2007) observaram aumento da produtividade com a realização da poda radicular, enquanto, WANG et al. (2014), observaram redução da produtividade de peras 'Clara Frijs' no primeiro ano da poda radicular, e aumento da produtividade na safra seguinte.

Quando foram avaliadas as características físicas e químicas dos frutos, observou-se que entre SS e $\mathrm{pH}$, não houve diferença significativa e que esta foi verificada somente para a variável firmeza de polpa (Tabela 2). Em estudo realizado por VERCAMMEN et al. (2005), os autores observaram aumento do conteúdo de sólidos solúveis com a realização da poda radicular, que pode ser explicado pela diminuição da 
translocação de água durante o amadurecimento. Os maiores valores para firmeza foram encontrados no tratamento testemunha e na poda radicular a $35 \mathrm{~cm}$ diferindo da poda radicular a $20 \mathrm{~cm}$. Nas plantas que receberam a poda radicular a $20 \mathrm{~cm}$ do lado direito observou-se menor firmeza de polpa. Normalmente, há uma relação inversamente proporcional entre firmeza de polpa e o tamanho do fruto, ou seja, quanto maior o fruto menor a firmeza de polpa, pela menor concentração de solutos na parede celular. A concentração de materiais de parede celular e a firmeza da polpa podem diminuir com o aumento do tamanho dos frutos, devido ao alongamento celular excessivo (TAIZ \& ZEIGER 2004).

Tabela 2. Variáveis físicas e químicas de frutos de pereira europeia 'Abate Fetel' com o uso de diferentes tipos de poda radicular na safra agrícola de 2007/2008.

Table 2. Physical and chemical variables of European pear fruits 'Abate Fetel' with the use of different types of root pruning in the 2007/2008 crop season.

\begin{tabular}{llll}
\hline Tratamento & $\mathrm{SS}\left({ }^{*}\right.$ Brix $)$ & $\mathrm{pH}$ & Firmeza de polpa $\left(\mathrm{kg} / \mathrm{cm}^{2}\right)$ \\
\hline Testemunha & $12,0 \mathrm{a}$ & $3,85 \mathrm{a}$ & $10,8 \mathrm{a}$ \\
$20 \mathrm{~cm}$ & $12,1 \mathrm{a}$ & $3,79 \mathrm{a}$ & $9,76 \mathrm{~b}$ \\
$35 \mathrm{~cm}$ & $12,5 \mathrm{a}$ & $3,85 \mathrm{a}$ & $11,1 \mathrm{a}$ \\
\hline CV $(\%)$ & 11,8 & 4,13 & 7,84 \\
\hline
\end{tabular}

Médias seguidas da mesma letra na coluna não apresentam diferença estatística pelo teste de Tukey a $5 \%$ de probabilidade de erro.

A poda radicular apresentou resultados positivos no controle do vigor e na manutenção de características vegetativas, não influenciando os índices produtivos e físico-químicos dos frutos. Conclui-se que a poda radicular promove controle do vigor em pereira europeia cultivar Abate Fetel, sendo portanto, uma alternativa para redução do excessivo crescimento vegetativo.

\section{REFERÊNCIAS}

ASÍN L et al. 2007. Effect of paclobutrazol, prohexadione-Ca, deficit irrigation, summer pruning and root pruning on shoot growth, yield, and return bloom, in a 'Blanquilla' pear orchard. Scientia Horticulturae 113: 142-148.

CARRA B et al. 2017a. Prohexadione calcium reduces vegetative growth and increases fruit set of 'Smith' pear trees, in Southern Brazil. Bragantia 76: 360-371.

CARRA B et al. 2017b. Control of the vegetative growth of 'shinsenki' pear trees by prohexadione calcium and root pruning. Pesquisa Agropecuária Brasileira 52: 177-185.

FACHINELLO JC \& ROBINSON TL 2017. Root pruning and prohexadione-calcium affect shoot growth of mature vertical axis apple trees. Acta Horticulturae 1177: 215-218.

FAO. 2016. Food and Agriculture Organization of the United Nations. FAOSTAT. Disponível em: http://faostat.fao.org/site/567/DesktopDefault.aspx?PagelD=567\#ancor. Acesso em: 18 jan. 2018.

HAWERROTH FJ et al. 2012. Redução da poda hibernal e aumento da produção de pereiras 'Hosui' pelo uso de prohexadiona cálcio. Pesquisa Agropecuária Brasileira 47: 939-947.

JANSSENS $P$ et al. 2011. Sensitivity of root pruned 'Conference' pear to water deficit in a temperate climate. Agricultural Water Management 99: 58-66.

MARSAL L et al. 2002. Regulated deficit irrigation and recti cation of irrigation scheduling in young pear trees: an evaluation based on vegetative and productive response. European Journal of Agronomy 17: 111-122.

PASA MS et al. 2011. Hábito de frutificação e produção de pereiras sobre diferentes porta-enxertos. Pesquisa Agropecuária Brasileira 46: 998-1005.

RADEMACHER W 2004. Chemical regulation of shoot growth in fruit trees. Acta Horticulturae 653: 29-32.

TAIZ L \& ZEIGER E. 2004. Fisiologia Vegetal. 3.ed. Porto Alegre: Artmed. 710p.

VERCAMMEN $\mathrm{J}$ et al. 2005. Root pruning: a valuable alternative to reduce the growth of 'Conference'. Acta Horticulturae 671: 533-537.

YEHIA T et al. 2014. Effect of root pruning on vegetative growth and leaf mineral content of le conte pear trees. World Journal of Agricultural Sciences 10: 253-260.

WANG $Y$ et al. 2014. Effect of root pruning and irrigation regimes on pear tree: growth, yield and yield components. Horticultural Science 41: 34-43. 